\title{
EMBEDDING THEOREMS AND QUASI-LINEAR ELLIPTIC BOUNDARY VALUE PROBLEMS FOR UNBOUNDED DOMAINS
}

\author{
BY \\ MELVYN S. BERGER AND MARTIN SCHECHTER( ${ }^{(1)}$
}

ABSTRACT. The Sobolev-Kondrachov embedding and compactness theorems are extended to cover general unbounded domains, by introducing appropriate weighted $L_{p}$ norms. These results are then applied to the Dirichlet problem for quasi-linear elliptic partial differential equations and isoperimetric variational problems defined on general unbounded domains in $\mathbf{R}^{N}$.

The general study of boundary value problems for quasi-linear elliptic partial differential equations has been generally limited to bounded domains. Perhaps one reason for this fact is that such compactness theorems as that of SobolevKondrachov and its extensions are no longer valid for general unbounded domains in $\mathbf{R}^{N}$. Consequently the degree theory of Leray-Schauder and the critical point theory based on such compactness properties as Condition (C) of Palais-Smale are not applicable in the study of quasi-linear elliptic problems defined on such general domains. In this article, we extend the Sobolev-Kondrachov compactness and embedding theorems to general unbounded domains and apply these results to quasi-linear Dirichlet problems and to nonquadratic isoperimetric variational problems. For quadratic isoperimetric problems some special embedding and compactness theorems of the type discussed here have been obtained recently ([1], [2], and [3]) in conjunction with the study of discrete spectra of linear elliptic partial differential operators of order $2 m$ defined on $\mathbf{R}^{\mathbf{N}}$. Our embedding theorems also extend some research of Gluško and Kreìn [4]. Some of the results presented here were announced by us in [0].

The present article is organized as follows: In $\$ 1$, we mention the types of elliptic boundary value problems to be discussed. In $\$ 2$, we state the $L_{p}$ embedding and compactness theorems that extend the results of Sobolev-Kondrachov. Applications of these theorems to quasi-linear elliptic boundary value problems are given in $\$ 3$. Finally, in $\S 4$, we prove the embedding theorems of $\$ 2$.

1. Quasi-linear elliptic problems on unbounded domains. Let $\Omega$ be an open set in $\mathbf{R}^{n}$ with boundary $\partial \Omega$. In this section we mention some problems that arise

Received by the editors June 16, 1971 and, in revised form, January 5, 1972.

AMS 1970 subject classifications. Primary $35 \mathrm{~J} 600$, 58E 15.

Key words and phrases. $L_{p}$ embedding, nonlinear eigenva lue problems, isoperimetric problems, critical point theory, quasi-linear elliptic boundary value problems.

(1) Research partially supported by grants from the NSF. 
in the study of quasi-linear elliptic partial differential operators of the form

defined on $\Omega$.

$$
A u=\sum_{|\alpha| \leq m}(-1)^{|a|} D^{\alpha}\left(a_{\alpha}\left(x, u, \cdots, D^{m} u\right)\right)
$$

(a) The Dirichlet problem. Let $f(x)$ be a smooth function defined on $\Omega$ and approaching a limit as $|x| \rightarrow \infty$. We seek a solution of

$$
A u=f \quad \text { in } \Omega ;\left.\quad D^{\alpha} u\right|_{\partial \Omega}=0, \quad|\alpha|<m,
$$

such that $D^{a} u \rightarrow 0$ as $|x| \rightarrow \infty$ for $|a|<m$. For bounded domains and coefficients $a_{a}\left(x, z_{\beta}\right)$ satisfying natural smoothness and growth conditions, the solvability of (1.1) is known to depend on a positivity condition on the form

$$
\mathfrak{U}_{m}\left(z_{\beta}\right)=\sum_{|\alpha|,|\gamma|=m ;|\beta|<m}\left\{a_{a}\left(x, z_{\beta}, z_{\gamma}\right)-a_{\alpha}\left(x, z_{\beta}, z_{\gamma}^{\prime}\right)\right\}\left(z_{\gamma}-z_{\gamma}^{\prime}\right)
$$

and a coerciveness condition on the form

$$
(A u, u)=\sum_{|\alpha| \leq m}\left(a_{\alpha}\left(x, u, \ldots, D^{m} u\right), D^{a} u\right)
$$

stating that

$$
(A u, u) /\|u\|_{m, p} \rightarrow \infty \text { as }\|u\|_{m, p} \rightarrow \infty, u \in \dot{W}^{m, p}(\Omega)
$$

(cf. [5]). In other words, as in the theory of linear elliptic equations, the presence of "small" lower order terms in $A$ does not alter the solvability of (1.1). It is well known in the linear case that if $\Omega$ is unbounded, the situation is quite different (cf., e.g., [6]). In particular, the notion of "smallness" must be reconsidered. Furthermore, applications require that the coefficients of $A$ be allowed to contain singularities while the solutions of (1.1) must be smooth.

(b) Isoperimetric variational problems. Given two smooth functionals

$$
\mathfrak{U}(u)=\int_{\Omega} F\left(x, u, \ldots, D^{m} u\right), \quad \mathfrak{B}(u)=\int_{\Omega} G\left(x, u, \ldots, D^{m-1} u\right),
$$

we seek critical points (in a suitable class $\mathcal{C}$ ) of $\mathcal{U}(u)$ subject to the constraint $\mathscr{B}(u)=$ constant, as $w e l l$ as critical points for the conjugate problem (i.e. critical points of $\mathscr{B}(u)$ subject to the constraint $\mathscr{A}(u)=$ constant). If $\mathcal{C}$ is the Sobolev space $\dot{W}^{m, p}(\Omega)$, the Euler-Lagrange equations for this problem give rise to a Dirichlet problem analogous to (1.1) of the form $A u=\lambda B u$, where $\lambda$ is a real parameter. If both functionals $\mathfrak{A}$ and $\mathscr{B}$ are quadratic, the critical points desired correspond to the spectral points of $A u=\lambda B u$. For unbounded domains, even if the quadratic functionals $\mathscr{A}$ and $B$ satisfy suitable ellipticity and positivity conditions, the proof of the existence of the spectral values is a difficult problem.

(c) Stationary states for nonlinear wave equations. Consider complex-valued solutions of the nonlinear wave equation

$$
v_{t t}=L v+f\left(x,|v|^{2}\right) v
$$


which are of the form $v(x, t)=e^{i \lambda t} u(x)$ subject to appropriate homogeneous boundary conditions on $\partial \Omega$. Here $\lambda$ is a real number, $u(x)$ is real-valued, and $L$ is a formally selfadjoint linear elliptic operator of order $2 m$. Such solutions are called stationary states. In the linear case they are of importance due to the principle of limiting amplitude [7] which gives conditions under which any solution $w(x, t)$ of (1.2) is asymptotic to a stationary state as $t \rightarrow \infty$ (i.e., $w(x, t)=e^{i \lambda t} u(x)+o(1)$ as $t \rightarrow \infty)$.

To summarize, many well-known problems have analogues for unbounded domains. However, in all cases the following problems must be overcome:

1. A substitute must be found for the Rellich-Kondrachov compactness theorem which is not valid for general unbounded domains.

2. For general unbounded domains $\Omega$ it does not follow that $q<p$ implies $L^{p}(\Omega) \subset L^{q}(\Omega)$.

3. One must find conditions under which $\dot{W}^{m, p}(\Omega)$ can be embedded in $W^{m-r, s}(\Omega)$.

2. The embedding theorems. Let $C_{0}^{\infty}$ denote the set of infinitely differentiable complex valued functions with compact supports in $\mathbf{R}^{N}$. For $\phi \in C_{0}^{\infty}$, s real, and $1<p<\infty$, we define the norm

$$
\|\phi\|_{s, p}=\left[\int\left|\vec{F}\left(1+|\xi|^{2}\right)^{s / 2} F \phi\right|^{p} d x\right]^{1 / p},
$$

where $F$ denotes the Fourier transform, $x=\left(x_{1}, \ldots, x_{n}\right)$ is a coordinate system in $\mathbf{R}^{n}, \xi=\left(\xi_{1}, \cdots, \xi_{n}\right)$ is the argument of $F \phi$ and $\bar{F}$ denotes the inverse Fourier transform. For $s$ a nonnegative integer, the norm (2.1) is equivalent to the sum of the $L^{p}\left(\mathbf{R}^{n}\right)$ norms of $\phi$ and all its derivatives up to order $s$ (cf. [8]). The completion of $C_{0}^{\infty}$ with respect to the norm (2.1) will be denoted by $H^{s, p}$.

Let $w(x)$ be a measurable function on $\mathbf{R}^{n}$. For $a>0$ and $1<p<\infty$ we set

$$
\begin{aligned}
\omega(x) & = \begin{cases}|x|^{\alpha-n} & \text { for } a<n, \\
1-\log |x| & \text { for } \alpha=n, \\
1 & \text { for } \alpha>n ;\end{cases} \\
M_{a, p, \delta, x}(w) & =\int_{|y|<\delta}|w(x-y)|^{p} \omega_{\alpha}(y) d y, \\
M_{a, p}(w) & =\sup _{x \in \mathbf{R}^{n}} M_{a, p, 1, x}(w), \\
N_{a, \delta}(w) & =\sup _{x}^{x} M_{a, 2, \delta, x}(w), \\
N_{\alpha}(w) & =N_{a, 1}(u) .
\end{aligned}
$$

We let $M_{a, p}$ [resp. $N_{a}$ ] denote the set of functions $w$ which satisfy $M_{a, p}(w)<\infty$ [resp. $\left.N_{a}(w)<\infty\right]$. Our first result is

Theorem 2.1. Assume that $s>0, q \geq p>1$ and $1 / q>1 / p-s / n$. Let $a>0$ 
be such that

$$
(\alpha-n) / q<s-n / p .
$$

Then there is a constant $C$ depending only on $p, q, s, n$ and such that

$$
\|w u\|_{0, q} \leq C M_{a, q}(w)^{1 / q}\|u\|_{s, p}, \quad u \in H^{s, p}, w \in M_{a, q^{*}}
$$

Theorem 2.2. Let $p, q, s, n$ and a satisfy the bypotbeses of Theorem 2.1. Suppose $w(x)$ is a function in $M_{a, q}$ sucb that

$$
\int_{|y|<1}|w(x-y)|^{q} d y \rightarrow 0 \text { as }|x| \rightarrow \infty .
$$

Then multiplication by $w$ is a compact operator from $H^{s, t}$ to $L^{q}$. Thus if $\left\{u_{k}\right\}$ is a sequence of functions in $H^{s, p}$ with uniformly bounded norms, then $\left\{w u_{k}\right\}$ bas a subsequence converging in $L^{q}\left(\mathbf{R}^{n}\right)$.

Let $\Omega$ be any domain in $\mathbf{R}^{n}$. For $s \geq 0$ we say that a function $u(x)$ on $\Omega$ is in $H^{s, p}(\Omega)$ if it is the restriction to $\Omega$ of a function in $H^{s, p}$. We set

$$
\|u\|_{s, p}^{s,}=\inf \|v\|_{s, p}, \quad v \in H^{s, p}, v=u \text { on } \Omega \text {. }
$$

It is easily checked that this is a norm and that $H^{s, p}(\Omega)$ is a Banach space. For functions $b(x)$ defined on $\Omega$, let $b_{\Omega}$ be given by

$$
b_{\Omega}(x)= \begin{cases}b(x) & \text { for } x \in \Omega, \\ 0 & \text { for } x \notin \Omega .\end{cases}
$$

We set $M_{a, p}(b, \Omega)=M_{a, p}\left(b_{\Omega}\right)$ and let $M_{a, p}(\Omega)$ denote the set of those functions $b$ on $\Omega$ such that $M_{a, p}(b, \Omega)<\infty$. Employing these definitions, we obtain

Theorem 2.3. Let $\Omega$ be any domain in $\mathbf{R}^{n}$. Under the bypotheses of Theorem 2.1,

$$
\|w u\|_{0, q}^{\Omega} \leq C M_{a, q}(w, \Omega)^{1 / q}\|u\|_{s, p}^{\Omega}, \quad u \in H^{s, p}(\Omega), w \in M_{a, q}(\Omega),
$$

where $C$ is the constant in (2.4).

Theorem 2.4. Under the bypotheses of Theorem 2.3, let $w$ be a function in $M_{\alpha, q}(\Omega)$ sucb that

$$
\int_{|x-y|<1 ; y \in \Omega}|w(y)|^{q} d y \rightarrow 0 \text { as }|x| \rightarrow \infty .
$$

Then multiplication by $w$ is a compact operator from $H^{s, p}(\Omega)$ to $L^{q}(\Omega)$.

As a specific application we note the following:

Theorem 2.5. Assume that $s>0, q \geq p>1$ and that $1 / q>1 / p-s / n$. Let $\alpha$ be a positive number satisfying (2.3). Then there is a constant $C$ sucb that

$$
\int_{\Omega} \frac{|u(x)|^{q}}{|x|^{\alpha}} d x \leq C\|u\|_{s, p}^{\Omega}, \quad u \in H^{s, p}(\Omega) .
$$


Moreover, if $\left\{u_{k}\right\}$ is a sequence of functions in $H^{s, p}(\Omega)$ with uniformly bounded norms, then $\left\{u_{k}(x) /|x|^{a / q}\right\}$ bas a subsequence converging in $L^{q}(\Omega)$.

The theorems given above also hold for the Sobloev spaces $W^{s, p}$ defined as follows. Take $\langle u\rangle_{0, p}=\|u\|_{0, p}$, and for $0<s<1$ set

$$
\langle u\rangle_{s, p}^{p}=\|u\|_{0, p}^{p}+\iint \frac{|u(x)-u(y)|^{p}}{|x-y|^{n+s p}} d x d y .
$$

For arbitrary $s \geq 0$ we let $k$ be the largest integer $\leq s$ and set

$$
\langle u\rangle_{s, p}=\|u\|_{k, p}+\sum\left\langle D^{k} u\right\rangle_{s-k, p},
$$

where $D^{k}$ denotes an arbitrary derivative of order $k$. One checks easily that $\langle u\rangle_{s, p}$ is a norm. The set of those functions $u \in L^{p}$ such that $\langle u\rangle_{s, p}<\infty$ is denoted by $W^{s, p}$. All of the theorems stated above hold with $H^{s, p}$ replaced by $W^{s, p}$. In particular we have

Theorem 2.6. Under the bypotheses of Theorem 2.1 there is a constant $C$ depending only on $p, q, s, n$ and a sucb th.,

$$
\|w u\|_{0, q} \leq C M_{a, q}(w)^{1 / q\langle u\rangle_{s, p}, \quad u \in W^{s, p}, . w \in M_{a, q}}
$$

If $w \in M_{a, q}$ and (2.3) bolds, then multiplication by $w$ is a compact operator from $W^{s, p}$ to $\stackrel{L^{q}}{q}$.

Let $\mu=\left(\mu_{1}, \cdots, \mu_{n}\right)$ be a multi-index of nonnegative integers. We set $|\mu|=$ $\mu_{1}+\cdots+\mu_{n}$ and $D^{\mu}=\partial|\mu| / \partial x_{1}^{\mu_{1}} \ldots \partial x_{n}^{\mu_{n}}$. Thus $D^{\mu}$ is a partial derivative of order $|\mu|$. For $k$ a nonnegative integer, we shall continue to let $D^{k}$ denote the generic derivative of order $k_{\text {. }}$

For $\Omega$ an arbitrary domain and $m$ a nonnegative integer, consider the norm

$$
\tilde{\| v}\left\|_{m, p}^{\Omega}=\sum_{|\mu| \leq m}\right\| D^{\mu} v \|_{0, p}^{\Omega}
$$

and let $\dot{W}^{m, p}(\Omega)$ [resp. $W^{m, p}(\Omega)$ ] denote the completion of $C_{0}^{\infty}(\Omega)$ [resp. $C^{\infty}(\bar{\Omega})$ ] with respect to this norm. Note that we can embed $\dot{W}^{m, p}(\Omega)$ continuously in $H^{m, p}(\Omega)$ and that the norms are equivalent. To see this, let $\phi$ be any function in $C_{0}^{\infty}(\Omega)$. Let $E \phi=\phi$ in $\Omega$ and $E \phi=0$ outside $\Omega$. Then $E \phi \in C_{0}^{\infty}$ and $\|E \phi\|_{m, p}$ is equivalent to $\widetilde{\|}\left\|_{m, p}=\widetilde{\| E} \phi\right\|_{m, p}^{\mathbf{R}^{n}}$. Now if $u$ is any function in $\dot{W}^{m, p}(\Omega)$, there is a sequence of functions $\left\{\phi_{k}\right\}$ in $C_{0}^{\infty}(\Omega)$ such that $\widetilde{\|} \phi_{k}-u \|_{m, p}^{\Omega} \rightarrow 0$. Thus $\left\{E \phi_{k}\right\}$ is a Cauchy sequence in $H^{m, p}$. Consequently, there is a $w \in H^{m, p}$ which is the limit of the $E \phi_{k}$. Clearly, $u=w$ on $\Omega$. Thus $u \in H^{m, p}(\Omega)$ and

$$
\|u\|_{m, p}^{\Omega \Omega} \leq\|w\|_{m, p}^{\Omega}=\lim \left\|E \phi_{k}\right\|_{m, p} \leq C \lim \widetilde{\|} \phi_{k}\left\|_{m, p}^{\Omega}=C \tilde{u}_{m}\right\|_{m, p}^{\Omega} .
$$

On the other hand, if $v \in H^{m, p}$ and $v=u$ on $\Omega$,

$$
\tilde{\|} u\left\|_{m, p}^{\mathbf{\Omega}}=\tilde{\|} w\right\|_{m, p}^{\mathbf{\Omega}} \leq \tilde{\|} w\left\|_{m, p}^{\mathbf{R}^{n}} \leq \tilde{\|}\right\|_{m, p}^{\mathbf{R}^{n}} \leq C\|v\|_{m, p} .
$$

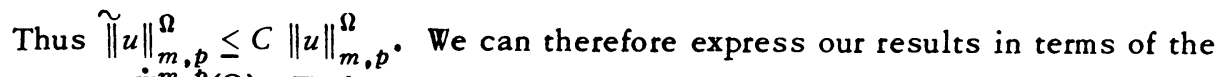
spaces $\dot{W}^{m, p}(\Omega)$. We have 
Theorem 2.7. Let $m$ be a positive integer. Assume that $q \geq p>1$ and that $1 / q>1 / p-m / n$. Let $\alpha>0$ be sucb that $(\alpha-n) / q<m-n / p$. Then there is $a$ constant $C$ depending only on $p, q, m, n$ and $a$ such that

$$
\|w u\|_{0, q}^{\Omega} \leq C M_{a, q}(w, \Omega)^{1 / q} \tilde{\|}_{u} \|_{m, p}^{\Omega}, \quad u \in \dot{W}^{m, p}(\Omega), w \in M_{a, q}(\Omega) .
$$

Moreover, if $w \in M_{a, q}(\Omega)$ satisfies (2.7), then multiplication by $w$ is a compact operator from $\dot{W}^{m, p}(\Omega)$ to $L^{q}(\Omega)$.

We now give an important application of Theorems 2.4 and 2.7. For each $x \in E^{n}$, let $S_{x}$ denote the ball of radius 1 having center at $x$.

Theorem 2.8. Assume that $1 / p-s / n<1 / q \leq 1 / p<1$. Then the embedding of $H^{s, p}(\Omega)$ into $L^{q}(\Omega)$ is compact if and only if the volume of $\Omega \cap S_{x}$ tends to 0 as $|x| \rightarrow \infty$. This is also a sufficient condition for the embedding $\dot{W}^{s, p}(\Omega)$ into $L^{q}(\Omega)$ to be compact.

3. Application of the embedding theorems to nonlinear problems. Consider the differential operator

$$
A u=\sum_{|a| \leq m}(-1)^{|a|} D^{\alpha}\left(a_{a}\left(x, u, \ldots, D^{m} u\right)\right) .
$$

We shall determine conditions on the functions $a_{a}$ so that $A$ is a bounded and. completely continuous operator on $W^{m, p}(\Omega)$. To this end, we consider the form

$$
F(\phi, u)=\sum_{|a| \leq m} \int_{\Omega} a_{a}\left(x, u, \cdots, D^{m} u\right) D^{a} \phi
$$

for $\phi \in C_{0}^{\infty}(\Omega)$ and $u \in \dot{W}^{m, p}(\Omega)$. We first give conditions which guarantee that for fixed $u \in \dot{W}^{m, p}(\Omega)$ the functional $F(\phi, u)$ will be bounded in $\phi$ with respect to the norm of $\dot{W}^{m, p}(\Omega)$. We then define the operator $\tilde{A}$ by

$$
F(\phi, u)=(\phi, \tilde{A} u), \quad \phi \in \dot{W}^{m, p}(\Omega),
$$

and then determine conditions such that $\widetilde{A}$ will be; (i) a bounded map from $\dot{W}^{m, p}(\Omega)$ to $W^{-m, p^{\prime}}(\Omega)$ and, (ii) map weakly convergent sequences into strongly convergent sequences. For these proofs the embedding theorems of $\$ 2$ are essential.

Throughout this section we let $p$ be a fixed number satisfying $1<p<\infty$ and for any multi-index $\mu$ we set

$$
1 / p_{\mu}=1 / p-(m-|\mu|) / n, \quad|\mu| \leq m,
$$

i.e. (by the Sobolev embedding theorem) $p_{\mu}$ is the largest number with $\dot{W}^{m, p}(\Omega) \subset$ $W^{|\mu|, p \mu}(\Omega)$ (the inclusion being both in the algebraic and topological sense). Our first assumption is

(i) The coefficients $a_{a}(x, z)$ with $z=\left(z_{\gamma_{1}}, z_{\gamma_{2}}, \cdots, z_{\gamma_{m}}\right)$ are measurable in $x$ and continuous in $z$ for almost all $x \in \Omega$ and 


$$
\left|a_{\alpha}(x, z)\right| \leq b_{\alpha}(x)+\sum_{|\gamma| \leq m} f_{a \gamma}(x)\left|z_{y}\right|^{\sigma(a, y)}
$$

where the $\sigma(\alpha, \gamma)$ are positive constants satisfying

$$
\sigma(\alpha, \gamma) \geq p-1
$$

and

$$
\sigma(\alpha, \gamma) / p_{\gamma}+1 / p_{a}<1
$$

(i.e. $\sigma(\alpha, \gamma)$ is so chosen that $|D u|^{\sigma(\alpha, \gamma)} \epsilon L^{p_{a}^{\prime}}$ with $\left.1 / p_{a}+1 / p_{a}^{\prime}=1\right), b_{a}(x) \epsilon$ $L^{p a}(\Omega)$ and $f_{a \gamma}(x) \in M_{s(a, \gamma), 1}(\Omega)$, where

$$
s(\alpha, \gamma)<n\left(1-\max \left[p / p_{\alpha}, \sigma(\alpha, \gamma) / p_{\gamma}+1 / p_{\alpha}\right]\right)
$$

(in order to insure for $u \in \dot{W}^{m, p}(\Omega)$ that $\int_{\Omega} f_{a \gamma}\left|D^{\gamma} u\right|<\infty$ ). Moreover, when $p_{\alpha}<0$ and $p_{y}>0$ we stipulate that

$$
\sigma(\alpha, \gamma)<p_{\gamma}
$$

and

$$
s(\alpha, \gamma)<n\left(1-(\max [p, \sigma(\alpha, \gamma)]) / p_{\gamma}\right)
$$

Similarly, when $p_{a}>0$ and $p_{y}<0$ we assume that

$$
s(\alpha, \gamma)<n\left(1-p / p_{\alpha}\right)
$$

Lemma 3.1. Under the above bypotheses, (I) there is a constant $C$ such that

$$
|F(\phi, u)| \leq C\|\phi\|_{m, p}\left(1+\sum_{a, \gamma}\|u\|_{m, p}^{\sigma(a, \gamma)}\right)
$$

and (II) the mapping $\widetilde{A}: \breve{W}^{m, p}(\Omega) \rightarrow W^{-m, p^{\prime}}(\Omega)$ (defined by (3.3)) is bounded.

Proof. Let $\alpha$ and $\gamma$ be fixed. We search for a number $t$ such that

$$
p \leq t, \quad 1 / p_{\alpha}<1 / t, \quad s(\alpha, \gamma)<n\left(1-t / p_{\alpha}\right),
$$

(3.14) $p \leq t^{\prime} \sigma(\alpha, \gamma), \quad \sigma(\alpha, \gamma) / p_{\gamma}<1 / t^{\prime}, \quad s(\alpha, \gamma)<n\left(1-t^{\prime} \sigma(\alpha, \gamma) / p_{\gamma}\right)$.

Once we have a $t$ satisfying (3.13) and (3.14) note that $\left[f_{a, y}(x)\right]^{1 / t}$ is in $M_{s(a, \gamma), t}(\Omega)$. Thus multiplication by this function is a bounded operator from $\dot{W}^{m-|a|, p}(\Omega)$ to $L^{t}(\Omega)$ (Theorem 2.7). Moreover, $\left[f_{a \gamma}(x)\right]^{1 / t^{\prime} \sigma(a, \gamma)}$ is in $M_{s(a, \gamma), t^{\prime} \sigma(a, \gamma)}(\Omega)$. Consequently, multiplication by this function is a bounded operator from $\dot{W}^{m-|\gamma|, p}(\Omega)$ to $L^{t^{\prime} \sigma(a, \gamma)}(\Omega)$. Hence

$$
\begin{aligned}
& \int_{\Omega} f_{a \gamma}\left|D^{\gamma} u\right|^{\sigma(a, \gamma)}\left|D^{\alpha} \phi\right| \leq\left\|f_{a \gamma}^{1 / t} D^{a} \phi\right\|_{0, t}\left\|f_{a \gamma}^{1 / t^{\prime}}\left|D^{\gamma} u\right|^{\sigma(a, \gamma)}\right\|_{0, t^{\prime}} \\
& \quad \leq C\left\|D^{a} \phi\right\|_{m-|a|_{, p}}\left\|f_{a \gamma}^{1 / t^{\prime} \sigma(a, \gamma)} D^{\gamma} u\right\|_{0, t^{\prime} \sigma(a, \gamma)}^{\sigma(a), \gamma} \leq C^{\prime}\|\phi\|_{m, p}\|u\|_{m, p}^{\sigma(a, \gamma)}
\end{aligned}
$$

Now by (3.2) and (3.12) 


$$
\begin{aligned}
|F(\phi, u)| & \leq \sum_{|\alpha|<m} \int_{\Omega}\left|a_{a}\left(x, u, \cdots, D^{m} u\right)\right|\left|D^{\alpha} \phi\right| \\
& \leq \sum_{|\alpha|<m}\left\|b_{a}\right\|_{0, p^{\prime}}\left\|D^{a} \phi\right\|_{0, p}+\sum_{|\alpha| \leq m ;|\gamma| \leq m} \int_{\Omega} f_{a \gamma}\left|D^{\gamma} u\right|^{\sigma(\alpha, \gamma)}\left|D^{\alpha} \phi\right| \\
& \leq C\left(1+\sum_{a, \gamma}\|u\|_{m, p}^{\sigma(a, \gamma)}\right)\|\phi\|_{m, p} .
\end{aligned}
$$

Now we show that under the hypotheses of the lemma we can find numbers $t$ and $t^{\prime}$ satisfying (3.13) and (3.14). When $p_{a}$ and $p_{y}$ are both positive, (3.3) and (3.4) are accomplished by setting $t=p$ when

$$
\sigma(\alpha, \gamma) / p_{\gamma}+1 / p_{\alpha} \leq p / p_{\alpha}
$$

and by setting

$$
t=p_{\alpha}\left(\sigma(\alpha, \gamma) / p_{\gamma}+1 / p_{\alpha}\right)
$$

otherwise. If $t=p$, then (3.13) follows. This proves the first part of the lemma.

By the first part of Lemma 3.1, we see that for fixed $u \in \dot{W}^{m, p}(\Omega)$, the expression $F(\phi, u)$ is a bounded linear functional on $\dot{W}^{m, p}(\Omega)$ with respect to $\phi$. Thus for each $u \in \dot{W}^{m, p}(\Omega)$ there is an element $\widetilde{A} u \in W^{-m, p^{\prime}}(\Omega)$ such that $F(\phi, u)=$ $(\phi, \widetilde{A} u)$. This gives a mapping $\widetilde{A}$ of $\dot{W}^{m, p}(\Omega)$ into $W^{-m, p^{\prime}}(\Omega)$ which is bounded, by Lemma 3.1 , i.e. $\widetilde{A}$ maps bounded sets in $\dot{W}^{m, p}(\Omega)$ into bounded sets in $W^{-m, p^{\prime}}(\Omega)$, from (3.8). Moreover, (3.15) implies $p^{\prime} \sigma(\alpha, \gamma) / p_{\gamma} \leq p / p_{\alpha^{*}}$. This inequality and (3.6) imply (3.14). If $t$ is given by (3.18), then (3.13) follows from (3.7) and (3.8). Also $t^{\prime} \sigma(\alpha, \gamma)=t \sigma(\alpha, \gamma) /(t-1)=t p_{\gamma} / p_{a^{*}}$. Thus (3.14) holds as well. When $p_{a}<0$ and $p_{y}>0$, we consider two cases. If $\sigma(\alpha, \gamma)<p$, we take $t=$ $p /(p-\sigma(\alpha, \gamma))$. Then $t^{\prime}=p / \sigma(\alpha, \gamma)$ and all of the inequalities in (3.13) and (3.14) are satisfied. If $p \leq \sigma(\alpha, \gamma)$, we take $t^{\prime}>1$ so close to 1 that $t^{\prime} \sigma(\alpha, \gamma)<p_{\gamma}$, $t \geq p$ and $s<n\left(1-t^{\prime} \sigma(\alpha, \gamma) / p_{\gamma}\right)$. Then all of the inequalities of (3.13) and (3.14) are satisfied. If $p_{a}>0$ and $p_{\gamma}<0$, take $t=p$. Then (3.13) and (3.14) are satisfied by (3.6) and (3.11). Do the same for case (d).

Remark. It follows from the results of [9] that the map $A$ is also continuous.

We now consider hypotheses on the differential operator

$$
\mathscr{R} u=\sum_{|\alpha| \leq m-1}(-1)^{|\alpha|} D^{a} b_{\alpha}\left(x, u, \cdots, D^{m-1} u\right)
$$

sufficient to insure the complete continuity of the associated abstract operator $\widetilde{\mathscr{P}}: \dot{W}^{m, p}(\Omega) \rightarrow W^{-m, p^{\prime}}(\Omega)$ defined in analogy with (3.2) by setting

$$
(\widetilde{\mathscr{R}} u, \phi)=\sum_{|\alpha| \leq m-1} \int_{\Omega} b_{\alpha}\left(x, u, \ldots, D^{m-1} u\right) D^{\alpha} \phi .
$$

In fact, we suppose that $b_{a}(x, \cdot)$ is measurable in $x$, while $b_{a}(x, 0) \in L^{p_{a}^{\prime}}(\Omega)$.

$$
\left|b_{\alpha}(x, z)-b_{\alpha}\left(x, z^{\prime}\right)\right| \leq \sum_{|\gamma| \leq m-1} f_{\alpha \gamma}(x)\left\{\left|z_{\gamma}\right|+\left|z_{\gamma}^{\prime}\right|\right\}^{\sigma(a, \gamma)-1}\left|z_{\gamma}-z_{\gamma}^{\prime}\right|
$$


where, as in (i), $\sigma(\alpha, \gamma)$ and $f_{a \gamma}$ satisfy the inequalities (3.6), (3.7) and (3.8) for $|\alpha|,|\gamma| \leq m-1$, and

$$
\int_{|x-y|<1 ; x \in \Omega} f_{a y}(y) d y \rightarrow 0 \text { as }|x| \rightarrow \infty .
$$

Clearly, Lemma 3.1 implies that the operator $\tilde{\mathscr{E}}$ is well defined and bounded since (ii) reduces to (i) when $z_{\gamma}^{\prime} \equiv 0$. Actually we shall prove

Lemma 3.2. The abstract operator $\tilde{\mathscr{B}}$ is completely continuous, i.e. $\tilde{\mathscr{B}}$ maps weakly convergent sequences in $\dot{W}^{m, p}(\Omega)$ into strongly convergent sequences in $W^{-m} \cdot p^{\prime}(\Omega)$, provided that the differential operator $\mathbb{B}$ satisfies bypotheses (ii) and (iii).

Proof. Let $u_{n} \rightarrow u$ weakly in $\dot{W}^{m, p}(\Omega)$, then we show that $\left\|\tilde{B} u_{n}-\tilde{B} u\right\| \rightarrow 0$ as $n \rightarrow \infty$. To this end, we estimate [with the definition (3.20)]

$$
\left(\tilde{\mathscr{B}}_{n}-\mathfrak{B} u, \phi\right)=\sum_{|\alpha| \leq m-1} \int_{\Omega}\left[b_{\alpha}\left(x, u_{n}, \cdots, D^{m-1} u_{n}\right)-b_{\alpha}\left(x, u, \cdots, D^{m-1} u\right)\right] D^{\alpha} \phi
$$

as follows. For fixed $|\gamma|,|\alpha| \leq m-1$,

$$
\begin{aligned}
& \left|\int_{\Omega}\left[b_{\alpha}\left(x, u_{n}, \cdots, D^{m-1} u_{n}\right)-b_{\alpha}\left(x, u, \cdots, D^{m-1} u\right)\right] D^{\alpha} \phi\right| \\
& \quad \leq \sum_{|\gamma| \leq m-1} \int_{\Omega} f_{\alpha \gamma}\left\{\left|D^{\gamma} u_{n}\right|+\left|D^{\gamma} u\right|\right\}^{\sigma(a, \gamma)-1}\left|D^{\gamma}\left(u_{n}-u\right)\right|\left|D^{\alpha} \phi\right| .
\end{aligned}
$$

Proceeding as in the proof of Lemma 3.1, with the numbers $t, t^{\prime}$ satisfying (3.13), (3.14), and by Hölder's inequality with $p=\sigma(\alpha, \gamma) /(\sigma(\alpha, \gamma)-1), q=\sigma(\alpha, \gamma)$,

$$
\begin{aligned}
\int_{\Omega} f_{a \gamma} & \left\{\left|D^{\gamma} u_{n}\right|+\left|D^{\gamma} u\right|\right\}^{\sigma(a, \gamma)-1}\left|D^{\gamma}\left(u_{n}-u\right)\right|\left|D^{\alpha} \phi\right| \\
\leq & \left\|f_{a \gamma}^{1 / t} D^{\alpha} \phi\right\|_{0, t}\left\|f_{a \gamma}^{1 / t^{\prime}}\left\{\left|D^{\gamma} u_{n}\right|+\left|D^{\gamma} u\right|\right\}^{\sigma(a, \gamma)-1} D^{\gamma}\left(u_{n}-u\right)\right\|_{0, t^{\prime}} \\
\leq & \left\|f_{a \gamma}^{1 / t} D^{\alpha} \phi\right\|_{0, t}\left\{\int_{\Omega} f_{a \gamma}\left[\left|D^{\gamma} u_{n}\right|+\left|D^{\gamma} u\right|\right]^{t^{\prime} \sigma(a, \gamma)}\right\} 1 / p \\
& \times\left\{\left.\left.\int_{\Omega} f_{a \gamma}\right|^{\gamma}\left(u_{n}-u\right)\right|^{t^{\prime} \sigma(a, \gamma)}\right\}_{1 / q} \\
\leq & \left\|f_{a \gamma}^{1 / t} D^{\alpha} \phi\right\|_{0, t}\left\|f_{a \gamma}^{1 / t^{\prime} \sigma(a, \gamma}\left\{\left|D^{\gamma} u_{n}\right|+\left|D^{\gamma} u\right|\right\}\right\|_{0, t^{\prime} \sigma(a, \gamma)}^{q / \phi} \\
& \times\left\|f_{a \gamma}^{1 / t^{\prime} \sigma(a, \gamma)} D^{\gamma}\left(u_{n}-u\right)\right\|_{t^{\prime} \sigma(a, \gamma)^{\prime}}^{t^{\prime}}
\end{aligned}
$$

Now as in the proof of Lemma 3.1, since $f_{a \gamma} \in M_{s(a, \gamma), 1}(\Omega), f_{a y}^{1 / \eta} \in M_{s(a, \gamma), \eta}(\Omega)$. Theorem 2.7 implies that multiplication by $f_{a y}^{1 / t}$ is a bounded (linear) map from $W^{m-|a|, p}(\Omega) \rightarrow L^{t}(\Omega)$, while multiplication by $f_{a y}^{1 / t^{\prime} \sigma(a, \gamma)}$ is a bounded (linear) map from $W^{m-|\gamma| \cdot p}(\Omega) \rightarrow L^{t^{\prime} \sigma_{(}}(\Omega)$. Theorem 2.8 implies $f_{a \gamma}^{1 / t^{\prime} \sigma\left(a_{0} \gamma\right)}$ is a compact map (which we denote $C^{a \gamma}$ ) from $W^{m-|\gamma|, p}(\Omega)$ into $L^{\sigma^{\prime}}(\Omega)$. Thus there are constants $K_{a \gamma}$, independent of $\phi$ and $u_{n}$, such that 


$$
\begin{aligned}
& \int_{\Omega} f_{a \gamma}\left\{\left|D^{\gamma} u_{n}\right|+\left|D^{\gamma} u\right|\right\}^{\sigma(a, \gamma)-1}\left|D^{\alpha} \phi\right| \\
& \leq K_{a \gamma}\left\|D^{\alpha} \phi\right\|_{m-|a|, p}\left\|D^{\gamma} u_{n}+D^{\gamma} u\right\|_{m-|\gamma|, p}^{q / p}\left\|C_{\alpha \gamma}\left(u_{n}-u\right)\right\|_{t^{\prime} \sigma(a, \gamma)}^{t^{\prime}} \\
& \leq K_{a \gamma}\|\phi\|_{m, p}\left(\left\|u_{n}\right\|_{m, p}+\|u\|_{m, p}\right)^{\sigma(a, \gamma)-1}\left\|C_{\alpha \gamma}\left(u_{n}-u\right)\right\|_{t^{\prime} \sigma(\alpha, \gamma)}^{t^{\prime}}
\end{aligned}
$$

Summing over $\alpha$, and noting that

$$
\begin{aligned}
\left\|B u_{n}-B_{u}\right\| & =\sup _{\|\phi\|=1}\left(B_{u_{n}}-B_{u, \phi}\right) \\
& \leq \sum_{a, \gamma} K_{a \gamma}\left(\left\|u_{n}\right\|_{m, p}+\|u\|_{m, p}\right)^{\sigma(a, \gamma)-1}\left\|C_{\alpha \gamma}\left(u_{n}-u\right)\right\|_{t^{\prime} \sigma(a, \gamma)^{\circ}}^{t^{\prime}}
\end{aligned}
$$

Thus, since $\left\{u_{n}\right\}$ is weakly convergent, there is a constant $M$ such that $\left\|u_{n}\right\|_{m, p} \leq$ $M$; so that as $n \rightarrow \infty,\left\|C_{a \gamma}\left(u_{n}-u\right)\right\| \rightarrow 0$ and $\left\|\widetilde{\mathscr{E}} u_{n}-\widetilde{\mathscr{E}} u\right\| \rightarrow 0$, as required.

As a simple first example of the boundedness and compactness theorems just proven, consider the following Dirichlet problem:

$$
\begin{aligned}
N u+B u & =f, \\
\left.D^{a} u\right|_{\partial \Omega} & =0, \quad|a| \leq m-1 .
\end{aligned}
$$

Here we suppose $N$ is a quasi-linear elliptic differential operator of the form

$$
N u=\sum_{|\alpha| \leq m}(-1)^{|\alpha|} D^{\alpha} N_{\alpha}\left(x, u, \cdots, D^{m} u\right)
$$

such that for $u \in \dot{W}^{m, p}(\Omega)$, with $N_{\alpha}(x, z)$ measurable in $x$, and continuous in $z$ (a.e. for $x, \in \Omega$ ),

$$
\sum_{|\alpha| \leq m} \int_{\Omega}\left\{N_{a}\left(x, D^{\gamma} u\right)-N\left(x, D^{\gamma} v\right)\right\} D^{\alpha}(u-v) \geq c\left(\|u-v\|_{m, p}\right)\|u-v\|,
$$

$$
\sum_{|a| \leq m} \int_{\Omega} N_{a}\left(x, D^{\gamma} u\right) D^{a} u \leq \phi\left(\|u\|_{m, p}\right)
$$

where $\phi(r)$ and $c(r)$ are finite real-valued functions of $r$ with $c(r) \rightarrow \infty$ as $r \rightarrow \infty$; and $B$ is a differential operator as described in Lemma 3.2, i.e. $B u=$ $\Sigma_{|a| \leq m-1}(-1)^{|a|} D^{a} B a_{a}\left(x, u, \cdots, D^{m-1} u\right)$. A simple example of an operator $N$ satisfying hypotheses (i) and (ii) is

$$
N u=\sum_{|a| \leq m}(-1)^{|a|} D^{\alpha}\left\{\left|D^{a} u\right|^{p-1} \operatorname{sgn} D^{a} u\right\} .
$$

We attempt to find a generalized solution of (3.21) by which we mean a function $u \in \dot{W}^{m, p}(\Omega)$ that satisfies the following integral identity for all $\phi \in C_{0}^{\infty}(\Omega)$ :

$$
\sum_{|a| \leq m} \int_{\Omega}\left[N_{a}\left(x, u, \cdots, D^{m} u\right)+B_{a}\left(x, u, \cdots, D^{m-1} u\right)\right] D^{\alpha} \phi=\int_{\Omega} f \phi .
$$

We shall prove the following result:

Theorem 3.2. Suppose the operator Bu satisfies the condition of Lemma 3.2, and in addition, that there is an absolute constant c such that, as $\|u\|_{m, p} \rightarrow \infty$, 


$$
\sum_{|\alpha| \leq m-1} \int_{\Omega}\left\{B_{\alpha}\left(x, u, \cdots, D^{m-1} u\right) D^{\alpha} u\right\} \geq-c\|u\|_{m, p}
$$

Then (3.21) bas a generalized solution for each $f \in W^{-m, q}(\Omega)$.

Proof. Lemma 3.1 above and standard results imply that generalized solutions of (3.21) are in (1-1) correspondence with solutions of an operator equation of the form

$$
\tilde{N} u+\widetilde{B} u=\tilde{f}
$$

where $\widetilde{N}$ and $\widetilde{B}$ are maps of $\dot{W}^{m, p}(\Omega) \rightarrow W^{-m, q}(\Omega)$ defined implicitly by the formulae

$$
\begin{aligned}
& (\tilde{N} u, \phi)=\sum_{|\alpha| \leq m} \int_{\Omega} N_{\alpha}\left(x, u, \cdots, D^{m} u\right) D^{\alpha} \phi, \\
& (\tilde{B} u, \phi)=\sum_{|\alpha| \leq m-1} \int_{\Omega} B_{\alpha}\left(x, u, \cdots, D^{m-1} u\right) D^{\alpha} \phi .
\end{aligned}
$$

By virtue of [5, Theorem 2.8] the operator $\tilde{N}$ is a homeomorphism of $\dot{W}^{m, p}(\Omega) \rightarrow$ $W^{-m, q}(\Omega)$ with $\|\widetilde{N} u\|_{-m, q} \geq c\|u\|_{m, p}$. Thus setting $v=\widetilde{N}^{-1} u$ the solutions of (3.22) are in (1-1) correspondence with the solutions of

$$
v+\bar{B} v=f
$$

where $\bar{B}=\widetilde{B} N^{-1}$ is a compact continuous map of $W^{-m, q}(\Omega)$ into itself. This last fact is, of course, an immediate consequence of Lemma 3.2 and the continuity of $N^{-1}$. Furthermore, (3.23) implies that all solutions $w$ of (3.24) satisfy the a priori estimate $\|w\|_{m, p} \leq K$. This follows since (3.23) can be rewritten as $(u, \widetilde{N} u+\widetilde{B} u) /\|\mu\| \rightarrow \infty$ as $\|u\|_{m, p} \rightarrow \infty$. Indeed if $\left\{w_{n}\right\}$ is à sequence of solutions of (3.24) with $\left\|w_{n}\right\|_{m, p} \rightarrow \infty$, one finds that

$$
\left(w_{n}, \widetilde{B} w_{n}\right) /\left\|w_{n}\right\|-c \leq\left(w_{n}, \tilde{N} w_{n}+\widetilde{B} w_{n}\right) /\left\|w_{n}\right\|_{m, p}
$$

$$
\leq\|f\|_{-m, q} \text { (a contradiction). }
$$

Consequently, all solutions $v$ of $(3.25)$ also satisfy an a priori bound of the form

$$
\|v\|_{-m, q} \leq K^{\prime}
$$

We are now in a position to apply the solvability theory centering around the LeraySchauder degree to (3.25). First (3.26) implies that for $K>\max \left(K^{\prime},\|f\|\right)$, equation (3.25) does not have a solution $v$ with $\|v\|=K$. Hence the Leray-Schauder degree $d\left(I+\bar{B}, f, S_{K}\right)$ is defined. Here $S_{K} \equiv\left\{v \mid v \in W^{-m, q},\|v\| \leq K\right\}$. We show $d\left(I+\bar{B}, f, S_{K}\right) \neq 0$, and consequently, by [10, pp. 102], $(I+\bar{B}) v=f$ is solvable in $S_{K}$, by proving that $d\left(I+\bar{B}, f, S_{K}\right)=d\left(I, f, S_{K}\right)=1$. To this end, by the homotopy invariance of degree, we need only show that the solutions of the equations $\tilde{N} u+$ $t \widetilde{B} u=f$ for $t \in[0,1]$ all lie in a fixed sphere in $W^{m, p}(\Omega)$. Indeed suppose there 
was a sequence of numbers $t_{n} \in[0,1]$ and elements $u_{n}$ with $\left\|u_{n}\right\| \rightarrow \infty$ such that $\widetilde{N} u_{n}+t_{n} B u_{n}=f$. Arguing as in (†), we find that (3.7) implies as, $\left\|u_{n}\right\| \rightarrow \infty$,

$$
\left(u_{n}, \widetilde{N} u_{n}\right) /\left\|u_{n}\right\|-c \leq\|f\|
$$

which contradicts hypothesis (i) on $\widetilde{N}$. Consequently, for $K$ sufficiently large, $d\left(I+K, f, S_{K}\right)=1$ so that (3.21) has a generalized solution as required.

Remark. The hypotheses of Theorem 3.2 can be weakened somewhat if one utilizes the theory of monotone mappings. Indeed we prove

Theorem 3.2'. Theorem 3.2 is still valid if the bypothesis (i) on the operator $\mathrm{Nu}$ is weakened to

$$
\sum_{|\alpha| \leq m} \int_{\Omega}\left[N_{\alpha}\left(x, D^{\gamma} u\right)-N_{\alpha}\left(x, D^{\gamma} v\right)\right] D^{\alpha}(u-v) \geq 0,
$$

$$
\sum_{|\alpha| \leq m} \int_{\Omega} N_{\alpha}\left(x, D^{\gamma} u\right) D^{\alpha} u \geq c\left(\|u\|_{m, p}\right)\|u\|_{m, p} .
$$

Proof. The result follows from the proof of $[10$, pp. 105-107, especially problem 3.8] or [5, p. 180, Theorem 2.7] by noting that if (i)' replaces (i) the resulting operator $T=\widetilde{N}+\widetilde{B}$ satisfies the properties (1) $\widetilde{N}$ is monotone and (2) $(T u, u) /\|u\|_{m, p}$ $\rightarrow \infty$ as $\|u\|_{m, p} \rightarrow \infty$ while (3) $\widetilde{B}$ maps weakly convergent sequences in $\dot{W}^{m, p}(\Omega)$ into strongly convergent sequences in $W^{-m, q}(\Omega)$.

Now we turn to a study of the isoperimetric problems mentioned in $\$ 1$. For $u \in \dot{W}^{m, p}(\Omega)$, we consider the critical points of the functional $\mathscr{U}(u)=$ $\int_{\Omega} N\left(x, u, \cdots, D^{m} u\right)$ subject to the constraint $B(u)=\int_{\Omega} G\left(x, u, \cdots, D^{m-1} u\right)$, provided of course that $\mathscr{U}(u), \mathfrak{B}(u)<\infty$ for each $u \in \dot{W}^{m, p}(\Omega)$. The associated Euler-Lagrange equation associated with this isoperimetric problem can be written

$$
\sum_{|\alpha| \leq m}(-1)^{|\alpha|} D^{\alpha} N_{\alpha}\left(x, u, \cdots, D^{m} u\right)=\lambda \sum_{|\beta| \leq m-1}(-1)^{|\beta|} D^{\beta} G_{\beta}\left(x, u, \cdots, D^{m-1} u\right),
$$

$$
\left.D^{\alpha}\right|_{\partial \Omega}=0, \quad|\beta| \leq m-1,
$$

where

$$
\begin{gathered}
N_{a}\left(x, z_{a_{1}}, \ldots, z_{a_{m}}\right)=\partial N\left(x, z_{a_{1}}, \ldots, z_{a_{m}}\right) / \partial z_{a} \\
G_{a}\left(x, z_{a_{1}}, \ldots, z_{a_{m-1}}\right)=\partial G\left(x, z_{a_{1}}, \cdots, z_{a_{m-1}}\right) / \partial z_{a}
\end{gathered}
$$

are locally Lipschitz continuous functions in the $\left\{z_{a}\right\}$ variables.

Theorem 3.3. Suppose the Fréchet derivative of $\mathscr{U}(u)$,

$$
\mathfrak{U}^{\prime}(u)=\sum_{|\alpha| \leq m}(-1)^{|\alpha|} D^{\alpha} N_{\alpha}\left(x, u, \cdots, D^{m} u\right) \text {, }
$$

satisfies the bypothesis mentioned at the beginning of Theorem 3.1, while $\mathfrak{P}^{\prime}(u)$ the Fréchet derivative of $\mathfrak{E}(u)$ satisfies

$$
\mathfrak{B}^{\prime}(u)=\sum_{|\alpha| \leq m-1}(-1)^{|\alpha|} D^{\alpha} G_{\alpha}\left(x, u, \cdots, D^{m-1} u\right)
$$


defines a completely continuous map $\tilde{\mathscr{B}}$ of $\dot{W}^{m, p}(\Omega) \rightarrow W^{-m, q}(\Omega)$ in accord with Lemma 3.1 such that $\widetilde{B}(0)=0$ and

$$
\sum_{|a| \leq m-1} \int_{\Omega} G_{a}\left(x, u, \cdots, D^{m-1} u\right) D^{a} u>0 \quad \text { for all } u \in \dot{W}^{m, p}(\Omega),
$$

then for each positive number $c$, inf $f_{(u)=c} \mathfrak{A}(u)$ is attained by some function $u_{c} \epsilon$ $\dot{W}^{m, p}(\Omega)$, and is a critical point of the function $\mathscr{U}(u)$ restricted to the surface $\mathfrak{B}(u)=c$. Furthermore, $u_{c}$ is a generalized solution of (3.25) for some $\lambda>0$.

Proof. By virtue of the arguments of Lemma 3.1 and Theorem 3.2, the result follows by invoking the following general result of [10].

Theorem. If $\nabla F(0)=0$ and $(\nabla F(u)-\nabla F(v), u-v) \geq k(\|u-v\|)\|u-v\|$ where $k(x) \rightarrow \infty$ as $|x| \rightarrow \infty$ while $\nabla G(u)$ is completely continuous with $(\nabla G(u), u)>0$ for $u \neq 0$, then inf $F(u)$ over the set $S_{c}=\{u \mid G(u)=c, c>0\}$ is attained by some $u_{c} \in S_{c}$ and $u_{c}$ satisfies $\nabla F\left(u_{c}\right)=\lambda \nabla G\left(u_{c}\right)$ for some $\lambda>0$.

Actually the result quoted is given only for Hilbert spaces, but the result easily generalizes to any reflexive Banach space. In the present case the formulae

and

$$
(\phi, F(u))=\sum_{|\alpha| \leq m} \int_{\Omega} N_{\alpha}\left(x, u, \cdots, D^{m} u\right) D^{\alpha} u
$$

$$
(\phi, G(u))=\sum_{|\alpha| \leq m-1} \int_{\Omega} G_{\alpha}\left(x, u, \cdots, D^{m-1} u\right) D^{\alpha} \phi
$$

implicitly define the maps $\nabla F$ and $\nabla G$ as operators from $\dot{W}^{m, p}(\Omega) \rightarrow W^{-m, q}(\Omega)$ as in (3.1).

More interesting results can be obtained for the conjugate variational problem, viz. the existence of critical points of the functional $\mathscr{B}(u)$ on the surface $\mathscr{U}(u)=$ constant in $\dot{W}^{m, p}(\Omega)$. To this end we prove

Theorem 3.4. Suppose the derivatives $\mathfrak{Q}^{\prime}(u)$ and $\mathfrak{B}^{\prime}(u)$ of the functionals $\mathscr{U}(u)$ and $\mathbb{B}(u)$ satisfy the bypotheses of Theorem 3.3, and are even in the variable $u$, then $\Re(u)$ bas a countably infinite number of critical points $\left\{u_{n}\right\}$ on the surface $\mathcal{S}_{c}=\{u \mid \mathcal{Q}(u)=$ const $>0\}$, these critical points satisfy (3.25) in the weak sense.

Proof. We identify antipodal points of $\delta_{c}$, and since the functional $B(u)$ is even, the critical points of $B(u)$ on $\delta_{c}$ correspond to the critical points of $\mathscr{R}(u)$ on $\delta_{c} / Z_{2}$. The set $\mathcal{S}_{c} / Z_{2} \subset \dot{W}^{m, p}(\Omega)$ has a natural Banach manifold structure and, in fact, is homeomorphic to $P^{\infty}\left(\dot{W}^{m}, p(\Omega)\right)$ the infinite-dimensional projective space defined on $\dot{W}^{m, p}(\Omega)$, defined by the canonical mapping of $\mathcal{S}_{c} \rightarrow \partial \Sigma_{1}=\{u \mid\|u\|=1\}$ along rays through the origin, see [11]. We now apply the Ljusternik-Schnirelmann theory of critical points to the functional $\{\mathscr{B}(u)\}^{-1}$ and the manifold $S_{c} / Z_{2}$. It follows by Palais [12] that since cat $P^{\infty}(X)=\infty$ for any Banach space $X,\{\mathscr{Q}(u)\}^{-1}$ 
will have a countably infinite number of distinct critical points on $\mathcal{S}_{c} / Z_{2}$ provided $\{B(u)\}^{-1}$ satisfies condition (C) on $\mathcal{S}_{c} / Z_{2}$.

To verify the Palais-Smale condition (C) in this case we take a sequence $u_{n} \epsilon$ $\delta_{c}$ such that $1 / \mathcal{B}(u)$ is bounded and show that if the gradient of the functional $1 / R\left(u_{n}\right)$ with respect to the manifold $\mathcal{S}_{c}$ tends to zero, then $\left\{u_{n}\right\}$ has a strongly convergent subsequence $\left\{u_{n_{j}}\right\}$. Indeed,

$$
\operatorname{grad} \delta_{c}\{1 / \mathscr{B}(u)\}=-(1 / \mathbb{B}(u))^{2} \operatorname{grad}_{\delta_{c}} \mathbb{B}(u)=-\left(1 / \mathbb{B}^{2}(u)\right)\{\nabla \mathfrak{B}(u)-\lambda \nabla A(u)\}
$$

where $\lambda=(\nabla B u, u) /(\nabla A u, u)$ and $\operatorname{grad}_{M}$ refer to the gradient of a functional relative to the manifold $M$. Thus if

$$
\left\|\operatorname{grad}_{S_{c}}\left\{1 / \mathscr{B}\left(u_{n}\right)\right\}\right\|=-\left(1 / \mathscr{B}^{2}\left(u_{n}\right)\right)\left\|\operatorname{grad}_{X} \mathfrak{B}\left(u_{n}\right)-\lambda_{n} \operatorname{grad}_{X} A\left(u_{n}\right)\right\| \rightarrow 0,
$$

$u_{n} \in \mathcal{S}_{c}$ is bounded in norm, and so has a weakly convergent subsequence $u_{n_{j}} \rightarrow$ $\bar{u}$ and $\operatorname{grad}_{X} \mathbb{B}\left(u_{n_{j}}\right) \rightarrow 0$. Hence, provided $\lambda_{n_{j}} \nrightarrow 0, \operatorname{grad}_{X} A\left(u_{n_{j}}\right)$ is strongly convergent. Thus by hypothesis, $u_{n_{j}} \rightarrow \bar{u}$ strongly and $\bar{u} \in \mathcal{S}_{c} / Z_{2}$ since this set is strongly closed. On the other hand, if $\lambda_{n_{j}}=\left(\nabla \mathbb{R}^{\prime} u_{n_{j}}, u_{n_{j}}\right) /\left(\nabla A^{\prime} u_{n_{j}}, u_{n_{j}}\right) \rightarrow 0$, the hypotheses on $\mathscr{U}(u)$ imply that $\left(\nabla \Re u_{n_{j}}, u_{n_{j}}\right) \rightarrow 0$, i.e. that since the functional $(\nabla B u, u)$ is weakly continuous and vanishes only if $u=0, u_{n_{j}} \rightarrow \bar{u}$ weakly so that $1 / B\left(u_{n_{j}}\right) \rightarrow 0$ since $B$ is weakly continuous. This contradicts the fact that $\left\{B\left(u_{n}\right)\right\}^{n}$ is uniformly bounded above zero.

This last theorem has many interesting consequences for the problems raised in $\$ \$ 1.3$ and 1.4. Let us consider the problems connected with the existence of stationary states. As mentioned in $\$ 1.3$, if we set $v=e^{i \lambda t} u(x)$ in equation (1.2), we find the following equation for $v$ and $\lambda$ :

$$
\begin{aligned}
\mathscr{L} u-\lambda^{2} u & =f\left(x,|u|^{2}\right) u, \\
\left.D^{\alpha} u\right|_{\partial \Omega} & =0, \quad|\alpha| \leq m-1 .
\end{aligned}
$$

Here

$$
\mathcal{L} u=\sum_{|\alpha|,|\beta| \leq m}(-1)^{|\alpha|} D^{\alpha}\left\{a_{\alpha \beta}(x) D^{\beta} u\right\}
$$

We shall prove

Corollary 3.5. Suppose the operator $\mathfrak{Q}$ possesses bounded, measurable coefficients $a_{a \beta}(x)$ and, for $u \in C_{0}^{\infty}(\Omega)$, the estimate

$$
(\mathcal{L}, u)=\sum_{|a|,|\beta| \leq m} \int_{\Omega} a_{\alpha \beta}(x) D^{a} u D^{\beta} u \geq \beta\left\{\sum_{|\alpha|=m} \int_{\Omega}\left|D^{\alpha} u\right|^{2}\right\}
$$

bolds for some absolute value $\beta>0$, while the function $f\left(x,|u|^{2}\right)$ is bomogeneous of degree $p>0$ with $f\left(x,|u|^{2}\right)|u|^{2}>0$ for $u \neq 0$. Then the equation $v_{t t}+\varrho_{v+}$ $f\left(x,|v|^{2}\right) v=0$ possesses a countably infinite number of stationary states of the form $v_{n}(x, t)=e^{i \lambda t} u_{n}(x)$ for eacb $\lambda \neq 0$ provided $f\left(x,|v|^{2}\right) \leq g(x)|v|^{\sigma}$ where 
$\sigma<4 m /(N-2 m)$ and $g(x) \rightarrow 0$ as $|x| \rightarrow \infty$.

Proof. The nontrivial solutions of (3.28) (after a change of scale) are the critical points of the functional $\int_{\Omega} F\left(x,|u|^{2}\right)$ where $F_{u}\left(x,|u|^{2}\right)=f\left(x,|u|^{2}\right) u$ subject to the constraint $\int_{\Omega}\left(L u \cdot u+\lambda^{2} u\right)=$ constant. Under the given hypotheses on $f\left(x,|u|^{2}\right)$, by virtue of Lemma 3.2, the nonlinear mapping $N: \dot{W}^{m, 2}(\Omega) \rightarrow W^{-m, 2}(\Omega)$ defined implicitly by the formula $(N u, v)=\int_{\Omega} f\left(x,|u|^{2}\right) u$ v is completely continuous. Thus by Theorem 3.4, there are an infinite number of distinct critical points $\tilde{u}_{n}$ for this variational problem. Hence after rescaling, these critical points give rise to the desired stationary states. The fact that these critical points are smooth enough to satisfy (3.28) pointwise is an immediate consequence of the $L_{p}$ regularity for linear elliptic differential equations as used in [13, pp. 168-172].

Corollary 3.5 can be considerably sharpened for the equation

$$
-i v_{t}=\Delta v+f\left(|v|^{2}\right) v
$$

provided one restricts attention to radially symmetric stationary states of the form $v(x, t)=e^{i \lambda t} u(|x|)$. Indeed, setting $|x|=r$ and $w(r)=r^{(N-1) / 2} u(|x|)$, the stationary states of (3.29) are determined by the nontrivial solutions of the following equation on $[0, \infty)$ :

$$
w_{r r}-\left(\lambda+(N-3)(N-1) / 4 r^{2}\right) w+f\left(r^{1-N} w^{2}\right) w=0, \quad w(0)=w(\infty)=0
$$

The conclusions of Theorem 3.4 thus apply to the system (3.30) provided $N>2$, $f(y) y^{2}>0$ for $y \neq 0$, and $|f(s)| \leq k|s|^{\sigma}$ with $0<\sigma<4 /(N-2)$. Consequently, under the above proviso, the conclusions of Corollary 3.5 hold for the system (3.29). Actually we prove

Corollary 3.6. For $N>1$, the system (3.29) possesses a countably infinite number of distinct radially symmetric stationary states $v_{n}(x, t)=e^{i \lambda t} u_{n}(|x|)$ for each $\lambda>0$ provided $f(s)$ is a Lipschitz continuous function (bomogeneous of degree $p>0)$ and sucb that $f(y) y^{2}>0$ for $y \neq 0$ with $|f(s)| \leq k|s|^{\sigma}$ for $0<\sigma<$ $4 /(N-2)$.

Proof. By the above remarks, it suffices to consider the case $N=2$. To this end we employ the following

Lemma 3.7. For $\lambda>0$, the operator $\ddot{w}-\left(\lambda-1 / 4 r^{2}\right) w$ bas discrete spectrum in $L_{2}(0, \infty)$ where $w(0)=0$.

(The proof of this lemma will be found in [14, pp. 127].)

In order to apply Theorem 3.4 to (3.14) in case $N=2$, we need only show that, for $\lambda>0$, there is an absolute constant $\beta>0$ such that for $w \in \dot{W}_{1,2}(0, \infty)$

$$
\int_{0}^{\infty}\left[w_{r}^{2}+\left(\lambda-\frac{1}{4 r^{2}}\right) w^{2}\right] d r \geq \beta \int_{0}^{\infty} w_{r}^{2} d r .
$$


The existence of the constant $\beta$ follows immediately from Lemma 3.7, standard results of the spectral theory of compact selfadjoint operators, and inequality

$$
\int_{0}^{\infty}\left[w_{r}^{2}+\left(\lambda-\frac{1}{4 r^{2}}\right) w^{2}\right] d r \geq \lambda^{2} \int_{0}^{\infty} w^{2} d r
$$

Here we have used the fact that for $w \in \dot{W}_{1,2}(0, \infty)$

$$
\int_{0}^{\infty} w_{r}^{2} d r \geq \frac{1}{4} \int_{0}^{\infty} \frac{w^{2}}{r^{2}} d r
$$

4. Proofs of the embedding theorems. In order to prove the theorems of $\$ 2$, we shall need the following lemmas.

Lemma 4.1. If $q \geq p>1, s>0$ and $1 / q>1 / p-s / n$, then there is a constant $C$ depending only on $p, q, s$ and $n$ such that

$$
\|u\|_{0, q} \leq C\|u\|_{s, p}, \quad u \in H^{s, p} .
$$

For a proof of this lemma see [15].

Lemma 4.2. If $q>1, s>0$ and $\alpha<q s$, then there is a constant $C$ depending only on $p, s, n$ and a sucb that

$$
\|v u\|_{0, q} \leq C M_{a, q}(v)^{1 / q}\|u\|_{s, q}, \quad v \in M_{a, q}, u \in H^{s, q} .
$$

Moreover, if $w \in M_{a_{0} q}$ and satisfies (2.5), then multiplication by $w$ is a compact operator from $H^{s, q}$ to $L^{q}$.

This lemma is proved in [3].

Lemma 4.3. For $s$ real, $p>1$ and $\epsilon>0$, there is a constant $C$ depending only on $s, p$ and $\epsilon$ such that

$$
\|u\|_{s, p} \leq C\langle u\rangle_{s+\epsilon, p}, \quad u \in C_{0}^{\infty} .
$$

For a proof see [17]. We can now give the

Proof of Theorem 2.1. Since (2.3) holds, there is a number $t$ such that

$$
a / q<t<s-n / p+n / q .
$$

In particular, $\alpha<q t$. Thus by Lemma 4.2 , there is a constant $C$ depending only on $q, t, \alpha$ and $n$ such that

$$
\|w u\|_{0, q} \leq C M_{a, q}(w)^{1 / q}\|u\|_{t, q}, \quad w \in M_{a, q}, u \in H^{t, q} .
$$

Consider the operator $G_{a} u=\bar{F}\left[\left(1+|\xi|^{2}\right)^{-a / 2} F u\right]$. By (2.1) for $b$ real and $p>1$ we have

$$
\left\|G_{a} u\right\|_{b, p}=\|u\|_{b-a, p} .
$$

By (4.4), $1 / q>1 / p-(s-t) / n$. In view of Lemma 4.1 , this implies

$$
\|u\|_{t, q}=\left\|G_{-t} u\right\|_{0, q} \leq C\left\|G_{-t} u\right\|_{s-t, p}=C\|u\|_{s, p} \text {. }
$$


Combining this with (4.2), we obtain (2.4).

Proof of Theorem 2.2. Let $t$ satisfy (4.4). By (4.7) the identity map is bounded from $H^{s, p}$ to $H^{t, q}$. By Lemma 4.2, multiplication by $w$ is a compact mapping from $H^{t, q}$ to $L^{q}$. This gives the result.

Proof of Theorem 2.3. Let $\epsilon>0$ and $u \in H^{s, p}(\Omega)$ be given. Then there is a $v \epsilon H^{s, p}$ such that $v=u$ on $\Omega$ and $\|v\|_{s, p}<\|u\|_{s, p}+\epsilon$. By Theorem 2.1,

$$
\left\|w_{\Omega} v\right\|_{0, q} \leq C M_{a, q}\left(w_{\Omega}\right)^{1 / q}\|v\|_{s, p} .
$$

since $w_{\Omega} v \in L^{q}$ and equals $w v$ on $\Omega$, this gives

$$
\|w u\|_{0, q}^{\Omega} \leq C M_{a, q}(w, \Omega)^{1 / q}\left(\|u\|_{s, p}+\epsilon\right)
$$

where $C$ does not depend on $\epsilon$. Letting $\epsilon \rightarrow 0$, we get (2.6).

Proof of Theorem 2.4. Let $\left\{u_{k}\right\}$ be a sequence of functions in $H^{s, p}(\Omega)$ such that $\left\|u_{k}\right\|_{s, p}^{\Omega} \leq N$. Then there is a sequence $\left\{v_{k}\right\}$ of functions in $H^{s, p}$ such that $v_{k}=u_{k}$ on $\Omega$ and $\left\|v_{k}\right\|_{s, p} \leq N+1$. Furthermore (2.7) is equivalent to

$$
\int_{|x-y|<1}\left|w_{\Omega}(y)\right|^{q} d y \rightarrow 0 \text { as }|x| \rightarrow \infty \text {. }
$$

Thus by Theorem 2.2, $\left\{w_{\Omega} v_{k}\right\}$ has a subsequence converging in $L^{q}$. This is the same as saying that $\left\{w u_{k}\right\}$ has a subsequence converging in $L^{q}(\Omega)$.

Proof of Theorem 2.5. Let $\beta$ be such that $\alpha<\beta<q(s-n / p+n / q)$. One checks easily that $w(x)=|x|^{-\alpha / q}$ is in $M_{\beta, q^{*}}$ Thus (2.8) follows from Theorem 2.3. Since $w(x) \rightarrow 0$ as $|x| \rightarrow \infty$, the remainder of the theorem follows from Theorem 2.4.

Proof of Theorem 2.6. There exists a $t<s$ such that $a<q(t p-n) / p$. By Lemma 4.3, $\|u\|_{t, p} \leq C^{\prime}\langle u\rangle_{s, p}$, and by Theorem $2.1\|w u\|_{0, q} \leq C M_{a, q}(w)^{1 / q}\|u\|_{t, p}$. This gives (2.9). If $w \in M_{a, q}$ satisfies (2.5) as well, the second conclusion follows from Theorem 2.2.

Proof of Theorem 2.7. The discussion preceding Theorem 2.7 shows that $\dot{W}^{m, p}(\Omega)$ can be continuously embedded in $H^{m, p}(\Omega)$ with equivalent norm. Thus Theorem 2.7 is an immediate consequence of Theorem 2.6.

Proof of Theorem 2.8. The sufficiency of the condition is immediate from Theorems 2.4 and 2.7. In fact, we have $w=1$ in this case, and (2.7) says

$$
\int|x-y|<1 ; y \in \Omega d y=\operatorname{Vol}\left(\Omega \cap S_{x}\right) \rightarrow 0 \text { as }|x| \rightarrow \infty \text {. }
$$

To prove the necessity, suppose there is a number $a>0$ and a sequence $\left\{x_{k}\right\}$ of vectors in $E^{n}$ such that $\left|x_{k}\right| \rightarrow \infty$ and

$$
\operatorname{Voi}\left(\Omega \cap S_{x_{k}}\right) \geq a, \quad k=1,2, \cdots
$$

Let $\phi(x)$ be a test function which is identically 1 on $S_{0}$, and set $\phi_{k}(x)=\phi\left(x-x_{k}\right)$, $k=1,2, \ldots$. Then $\phi_{k}$ equals 1 on $S_{x_{k}},\left\|\phi_{k}\right\|_{s, p}^{\Omega} \leq\left\|\phi_{k}\right\|_{s, p}=\|\phi\|_{s, p}$, and $\phi_{k}(x) \rightarrow 0$ as $k \rightarrow \infty$ for each $x \in E^{n}$. But we have 


$$
\left\|\phi_{k}\right\|_{0, q}^{q}=\int\left|\phi_{k}(x)\right|^{q} d x \geq a, \quad k=1,2, \cdots
$$

Thus the embedding of $H^{s, p}(\Omega)$ into $L^{q}(\Omega)$ cannot be compact. The statement concerning $W^{s, p}(\Omega)$ is an immediate consequence of the discussion preceding Theorem 2.7.

\section{BIBLIOGRAPHY}

0. M. S. Berger and M. Schechter, $L_{p}$ embedding and nonlinear eigenvalue problems for unbounded domains, Bull. Amer. Math. Soc. 76 (1970), 1299-1302: MR 42 \#2294.

1. A. M. Molcanov, On conditions for discreteness of the spectrum of self-adjoint differential equations of the second order, Trudy Moskov. Mat. Obšč. 2 (1953), 169-199. (Russian) MR 15, 224.

2. M. S. Birman, On the spectrum of singular boundary-value problems, Mat. Sb. 55 (97) (1961), 125-174; English transl., Amer. Math. Soc. Transl. (2) 53 (1966), 23-80. MR $26 \# 463$.

3. M. Schechter, $O_{n}$ the essential spectrum of an elliptic operator perturbed by a potential, J. Analyse Math. 22 (1969), 87-115. MR 40 \#579.

4. V. P. Gluško and S. G. Krě̃n, Inequalities for the norms of derivatives in weighted $L_{p}$ spaces, Sibirsk. Mat. Ž. 1 (1960), 343-382; English transl., Amer. Math. Soc. Transl. (2) 85 (1969), 1-50. MR \#A3507.

5. J. L. Lions, Quelques méthodes de résolution des problèmes aux limites nonlinéaires, Dunod; Gauthier-Villars, Paris, 1969. MR 41 \#4326.

6. B. R. Vaŭnberg, On elliptic problems in unbounded domains, Mat. Sb. 75 (117) (1968), 454-480 = Math. USSR Sb. 4 (1968), 419-444. MR 37 \#6601.

7. D. M. Ėudus, The principle of limiting amplitude, Uspehi Mat. Nauk 24 (1969), no. 3 (147), 91-156= Russian Math. Surveys 24 (1969), no. 3, 97-168.

8. L. Bers, F. John and M. Schechter, Partial differential equations, Lectures in Appl. Math., vol. 3, Interscience, New York, 1964. MR 29 \#346.

9. M. Vainberg, Variational methods for the study of non-linear operators, GITTL, Moscow, 1956; English transl., Holden-Day, San Francisco, Calif., 1964. MR 19, 567; MR $31 \# 638$.

10. M. S. Berger and M. Berger, Perspectives in nonlinearity. An introduction to nonlinear analysis, Benjamin, New York, 1968. MR 40 \#4971. 1969.

11. J. T. Schwartz, Nonlinear functional analysis, Gordon and Breach, New York,

12. R. Palais, Ljusternik-Schnirelman theory on Banach manifolds, Topology 5 (1966), 115-132. MR $41 \# 4584$.

13. M. S. Berger, An eigenvalue problem for nonlinear elliptic partial differential equations, Trans. Amer. Math. Soc. 120 (1965), 145-184. MR 31 \#6047.

14. E. C. Titchmarsh, Eigenfunction expansions associated with second-order differential equations. Part I, 2nd ed., Clarendon Press, Oxford, 1962. MR 31 \#426.

15. N. Aronszajn and K. T. Smith, The ory of Bessel potentials. I, Ann. Inst. Fourier (Grenoble) 11 (1961), 385-475. MR $26 \# 1485$. 1971.

16. M. Schechter, Spectra of partial differential operators, North-Holland, Amsterdam,

17. J. L. Lions and E. Magenes, Problemi ai limiti non omogenei. III, Ann. Scuola Norm. Sup. Pisa (3) 15 (1961), 41-103. MR $26 \# 4048$.

DEPARTMENT OF MATHEMATICS, BELFER GRADUATE SCHOOL OF SCIENCE, YESHIVA UNIVERSITY, NEW YORK, NEW YORK 10033 (Current address of Martin Schechter)

Current address (M. S. Berger): School of Mathematics, Institute for Advanced Study, Princeton, New Jersey 08540 